\title{
Nursing and Technology Foresight in Futures of a Complex World
}

\author{
Mohammad Reza Dawoudi* \\ Turku University of Applied Sciences, Finland \\ *Corresponding author: Mohammad Reza Dawoudi, Finland \\ Submission: 制 August 24, 2017; Published: 制 September 06, 2017
}

\begin{abstract}
Medical technology is one of the foundations health care systems in the world. Patient care technology has become increasingly complex and medical technology has improved health care efficiency, quality safety and cost. However, it is shown in patient care centers that current technology alone is not enough. Up to now several tools were originally created for morbid monitoring e.g. pain scale and bedsores protection to help patients communicate about their pain. However, in spite of the importance and prevalence of pain, there are currently no clinically accepted tools to objectively monitor changes in pain level, requiring physicians to rely on patients' subjective assessment, or to simply guess, when patients cannot describe their pain [1]. This article aimed to provide the theoretic framework for improving patient safety system, and enhances the ability of health care professionals to coordinate care by providing a patient's health information. This framework is based on Morbid-Monitoring Systems include "Pain and Unconsciousness Scales- measurements" and "Thermal Wound Injury Prevention System (TWIP)". The multi-methods approaches to monitor these processes are Morbid Motion Monitoring, Facial expressions and effective Thermal Radiation. This article identifies these novel technologies that change the practice of nursing, and explores issues on how these novel technologies are driving change in the nursing environment.
\end{abstract}

Keywords: Nursing informatics; Morbid monitoring; Pain scale; Bedsores; Thermal radiation

\section{Introduction}

Computer technology gives us a tool which can be used to help us cope with the complexity and efficiency which is often required in many areas of work [2]. Since the earliest days of technology adoption in health care, nursing informatics professionals have been at the forefront of leading change. Early pioneers included nurses who effectively combined the science of nursing with computer and information science to support the clinical workflow, adding value to the organization as they began their journey to join the digital revolution Mark [3] However, it has been difficult to find computer solutions that offer benefit to operational nurses, midwives and health visitors. Solutions are required that support patient orientated developments in practice [4]. Therefore in the future, the nursing informatics systems committees must be able to support quality morbid monitoring. This document provides the context for an examination of the issue of developing a nursing informatics strategy at the local level of patient care centers.

\section{Morbid Monitoring Systems}

Morbid monitoring can be defined as "repeated or continuous observations or measurements of the patient, his or her physiological function, and the function of life support equipment, for the purpose of guiding management decisions, including when to make therapeutic interventions, and assessment of those interventions" (Hudson, 1985, p. 630). Morbid monitoring system is classified by target parameter, e.g. monitoring treatment for chronic pain [5], painful monitoring, [6] pain management medication monitoring, [7] neurological monitoring which generally refers to electroencephalography, intracranial pressure, anesthesia and pain monitoring, coma monitoring, monitoring of wound, etc. However, up to now the goal of monitoring has been to measure the degree of injury and to prevent further injury, rather than to measure.

Sankalp [8] "Pain scales measurements", "Unconsciousness scale measurements" or "Injury Prevention". Up to now several tools were originally created for patients such as the Brief Pain Inventory, PEG tool, Wong-Baker FACES Pain Rating Scale, 0-10 Numeric Pain Rating Scale, Pain Assessment in Advanced Dementia Scale (PAINAD) and etc. to help them communicate about their pain. However, in spite of the importance and prevalence of pain, there are currently no clinically accepted tools to objectively monitor changes in pain level, requiring physicians to rely on patients' subjective assessment, or to simply guess, when patients cannot describe their pain [9].

The latest development in patient monitoring system can be used in Intensive Care Unit (ICU), Critical Care Unit (CCU), and Emergency Rooms of hospital. During treatment, the patient monitor is continuously monitoring the coma (a deep state of unconsciousness) patient to transmit the important information. 
Koganti [10] In 2011 Kansal and Dhillon have developed Advanced Coma Patient Monitoring System which is advanced product related to physical changes in body movement of the patient and gives Warning in form of alarm and display on the Liquid crystal display (LCD). It also passes a SMS to a person sitting at the distant place if there exists any movement in any body part of the patient [11]. In 2013 Sneha Chowdary and et al proposed the Wearable Motion sensor system, which is used to monitor the body movements such as eye blink movement and hand movement to detect the conscious state of an individual. This system will be helpful in assisting the doctor about the health condition of the uncon- scious patient and alerting the doctor whenever cares is required [12].

However, these methods did not address the management of coma patients injured by penetrating objects such as thermal radiation, pressure ulcer and stab wounds. Pressure ulcers occur because of soft tissue distortion, and incidence rates are on the rise in the healthcare arena (James G Spahn, 215). The pressure ulcer prevention protocol consisted of preventive interventions stratified on risk level, with implementation of support surfaces and turning/repositioning residents [13]. In 2013 Matthew and Pompeo have trialed new pressure map- ping technology for patients with pressure ulcers. The system contains thousands of sensors, which sensors display specific areas of pressure, and it provides live feedback to clinicians as they reposition patients [14]. However, for implementation of the system, the nursing staff needs to understand its ability to record data [15]. To overcome these significant problems, we have designed and developed an MorbidMonitoring System, with a novel technique to optimize the "pain and Unconsciousness scale measurements" and the prevention of "Thermal Wound Injury" and "pressure ulcers". The goal of this study is to develop Clinical Decision Support Systems for, "pain, unconsciousness and wound prevention managements that could be used by health care professionals throughout the world.

\section{Methods and Materials}

Pain and Unconsciousness Scale Measurements Two methods have described for the measurement of pain rate: Morbid mobility monitoring and facial expressions and Micro expression.

\section{Morbid mobility monitoring}

Motion of morbid has been defined as the rate of pine and it is linked with various comorbid conditions. The data which is generated by the hospital on the basis of video data recorder is separated frame by frame. The next set of function is about the visual differences between various frames. A variety of techniques can be used as a basis for comparison between frames. One of these techniques is called Texture analysis based on DNA Modeling. The method is based on the linear mapping and the one-to-one correspondences between point features extracted from the frames and on calculating similarities in pixel values. This correspondence is determined by comparing two strings constructed from pixel values of the frames. The method uses a table called the Quarter Code table, which is the set of characters and numbers. In this table every number between 0 and 255 is translated into a unique string of four letter alphabet. Letters A, C, G, T are chosen, since they are the same as used in DNA sequences. In this way it possible to utilize tools originally programmed to DNA sequences analysis. When all pixel values of frames (images) are converted to virtual DNA sequences, one can show the differences between two virtual DNA sequences. The com- parison between two virtual DNA sequences is done by Chi- squared test, DNA Sequence Alignment Algorithms (Needleman Wunsch and Smith Waterman), Markov Chain and glm plot. The rate of similarity between frames is plotted as a graph and it's appearing in Monitor. The system is used to show and describe the displacement of the morbid on the bedside. The amount of ill transmitted to the morbid increase the motion ratio. In this manner Motion Ration is the ratio of the displacement of the morbid.

\section{Facial expressions: interpretation of facial pain signals}

Neuropsychological and neuroimaging evidence suggests that the human brain contains facial expression recognition detec- tors specialized for specific discrete emotions [16]. Emotional facial expressions represent facial displays of emotions which determine different patterns of muscular correlates, cognitive responses, and brain activation [17]. Behavioural/facial markers of pain refer to a variety of responses that typically accompany the experience of pain. They serve the purpose to communicate the inner state "pain" to others and thus play a crucial role in social interactions [18]. Methods designed to capture and measure this dynamic phenomenon have greatly improved our understanding of discrete muscle action associated with the various facial displays [19]. In this study, a nov- el facial expression recognition method is proposed. The technique is based on DNA Modelling of digital images and their statistical analysis. This method is executed by ChiSquared test. ChiSquared test is a statistical test method commonly used to compare observed data with data we would expect.

\section{Prevention of Thermal Wound Injury and Pressure Ul- cers}

A pressure ulcer is a localized injury to the skin or underlying tissue, usually over a bony prominence, as a result of unre- lieved pressure. Pressure ulcers are caused by unrelieved pressure, applied with great force over a short period (or with less force over a longer period), that disrupts blood supply to the capillary network, impeding blood flow and depriving tissues of oxygen and nutrients. This external pressure must be greater than arterial capillary pressure to lead to inflow impairment and resultant local ischemia and tissue damage.

In 2015 Chaves and et al presented a study that suggest- ed a relationship between the temperature and area of pres- sure ulcers and proposed thermography as an adjunctive method for the evaluation of healing processes. In 2016 Bennett and colleagues began an examination of at-risk older adult patient data (pressure and thermal) to identify patterns that may related to pressure ulcer development. The results of this study suggested that a relationship may exist between the sleeping behavior of patient and the long term temperature distribution of the feet. However, before of these studies in 2013 Matthew Q. and Pompeo have trialed new pressure. 
Mapping technology for patients with pressure ulcers. In this technique, the device (The MAP System, Wellsense Inc, Nashville, $\mathrm{TN}$ ) is a thin mat placed on a mattress that has a color monitor attached. The mat contains thousands of sensors, and is secured to the top of the mattress with straps. These sensors measure pressures through a sensing area measuring $1945 \mathrm{~mm}$ x $805 \mathrm{~mm}$. The sensors display specific areas of pressure, and the system provides live feedback to clinicians as they reposition patients. The monitor acts as an educational tool for staff, patients, and family by indicating where the pressure points are located. It can also assist clini- cians in optimal surface selection and detection of malfunctioning mattresses. Other features include a bed alarm that can be set to sound at the desired interval to alert for turning.

\section{Hospital Application Integration}

Hospital Application Integration (HAI) is an integration framework composed of a collection of technologies and services to integrate a set of hospital computer applications. Hospital Computer Applications (HCA) is computer software used to integrate a set of hospital computer applications (Figure 1).

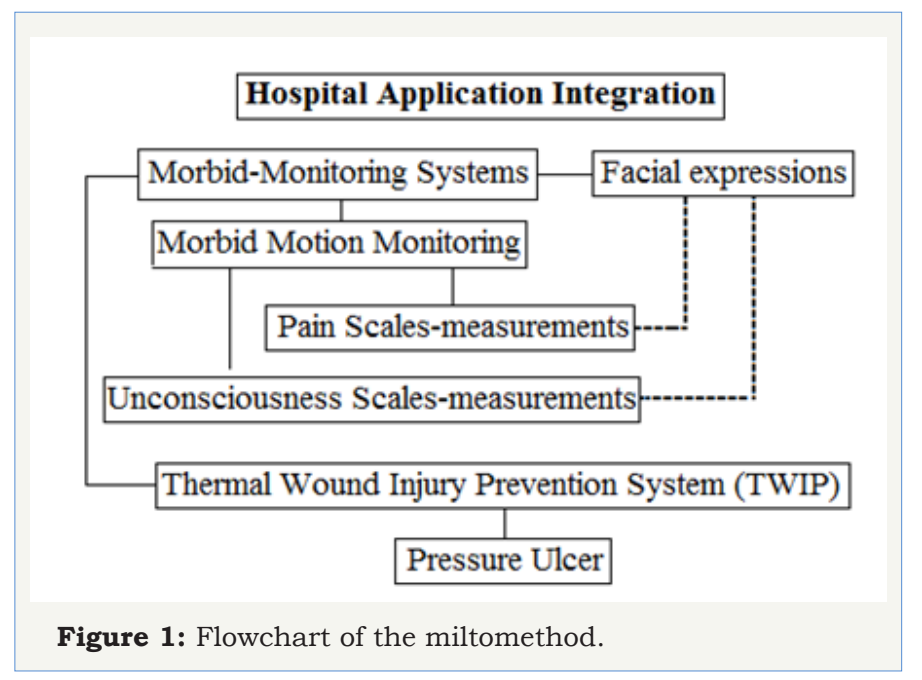

\section{Conclusion}

With the help of this system nurses can monitor the pain and unconsciousness scales with help of motion detection techniques and facial expressions. Nurses can also identify and prevent risk factors associated with hospital acquired preexisting pressure. Consequently, the system can enhance the ability of health care professionals to coordinate care by providing a patient's health information.

\section{References}

1. http://www.medasense.com/

2. Toromanovic S, Hasanovic E, Masic I (2010) Nursing Information Systems. Mater Sociomed 22(3): 168-171.

3. Sugrue MD (2013) Technology, transformation, and the nursing workforce. American Nurse Today, USA, 8(11).

4. https://www.nps.org.au/

5. Luceya P, Cohnb JF, Prkachind KM, Solomone PE, Chewf S, et al. (2012) Painful monitoring: Automatic pain monitoring using the UNBCMcMaster shoulder pain expression archive database. Image and Vision Computing 30(3): 197-205.

6. (2016) Pain Management Medication Monitoring. Drugscan, PA.

7. Gardner RM, Michaelshabot M (2016) Patient Monitoring Systems, pp. 585-625.

8. Sankalp M (2011) Coma Patient Monitoring System Using Image Processing. AIP Conference Proceedings 1414(1).

9. Kansal N, Dhillon HS (2011) Advanced Coma Patient Monitoring System. International Journal of Scientific \& Engineering Research 2(6): 22295518.

10. Koganti SC, Suma HN, Abhishek AM (2013) Analysis and Monitoring of Coma Patients using Wearable Motion Sensor System. International Journal of Science and Research, p. 4438.

11. Lyder CH, Ayello EA (2008) Pressure Ulcers: A Patient Safety Issue. In: Hughes RG, (Ed.), Patient Safety and Quality: An Evidence-Based Handbook for Nurses, Chapter 12, Virginia.

12.Pompeo MQ (2013) Pressure map technology for pressure ulcer patients: can we handle the truth? Wounds 25(2): 34-40.

13.Susskind JM, Littlewort G, Bartlett MS, Movellan J, Anderson AK, et al. (2007) Human and computer recognition of facial expressions of emotion. Neuropsychologia 45(1): 152-162.

14.Balconi M (2012) Consciousness and Emotion: Attentive vs. Preattentive Elaboration of Face Processing. Encyclopedia of the Sciences of Learning, pp. 774-776.

15. Miriam Kunz (2015) Behavioural/Facial Markers of Pain, Emotion, Cognition. Pain, Emotion and Cognition, pp. 123-133.

16. Katsikitis M (2003) Facem: The Facial Expression Measurement System. The Human Face, pp. 99-118.

17. Bluestein D, Javaheri A (2008) Pressure Ulcers: Prevention, Evaluation, and Management. Am Fam Physician 78(10): 1186-1194.

18. Abreu Chaves ME, da Silva FS, Campos Soares VP, Magalhães Ferreira RA, Latini Gomes FS, et al. (2015) Evaluation of healing of pressure ulcers through thermography: a preliminary study. Res Biomed Eng 31(1): 3-9.

19. Bennett SL, Goubran R, Knoefel F (2016) Measurement and comparison of thermal and in-bed pressure data towards the prevention of pressure ulcers. Biomedical and Health Informatics (BHI), 2016 IEEE-EMBS International Conference. 